\title{
Sarcoma fibromixoide intracraneal primario de bajo grado: reporte de caso y revisión de la literatura.
}

\author{
Low-grade fibro-myxoid sarcoma of intracranial origin: A case report and literature review. \\ José L. León-Palacios ${ }^{1,2, a}$, Augusto M. Palacios M. 1,a, Sandro Casavilca-Zambrano ${ }^{3,4, b, c}$,

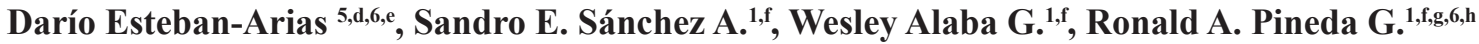

\section{RESUMEN}

El sarcoma fibromixoide de bajo grado (LGFMS) es un tumor de tejidos blandos de origen mesenquimal. Los sarcomas son un grupo heterogéneo, que representa el 1\% de todas las neoplasias. Los sarcomas primarios del sistema nervioso central (SNC) son raros y representan solo el $0,7 \%$ del total de sarcomas, con una incidencia estimada de 3 por cada 10 millones de personas por año. En este artículo, se describe el caso de una mujer de 59 años que presentó un sarcoma fibromixoide intracraneal de bajo grado, localizado en la región parietal derecha. Se discute el curso clínico, estudios de imágenes, características histopatológicas y tratamiento de este diagnóstico infrecuente y, por lo mismo, muy poco reportado. El diagnóstico definitivo se obtiene, ciertamente, mediante estudios histo- patológicos.

PALABRAS CLAVE: Sarcoma, neoplasias cerebrales, inmunohistoquímica.

\section{SUMMARY}

Low-grade fibromyxoid sarcoma (LGFMS) is a soft tissue tumor of mesenchymal origin. Sarcomas are a heterogeneous group, representing $1 \%$ of all neoplasm diagnoses. Primary sarcomas of the central nervous system (CNS) are rare, and represent only $0.7 \%$ of all sarcomas, with an estimated incidence of 3 per 10 million people per year. The case of a 59-year-old woman who developed a low-grade intracranial fibromyxoid sarcoma in the right parietal region, is described. The clinical course, imaging studies, histopathological features, and treatment approach of this unusual diagnosis, are discussed. Low-grade intracranial fibromyxoid sarcoma is a rare and probably underreported condition. The definitive diagnosis is usually made through histo-pathological studies.

KEY WORDS: Sarcoma, brain neoplasms, inmuno-histochemistry.

Departamento de Cirugía, Servicio de Neurocirugía, Hospital Cayetano Heredia, Lima, Perú.

Universidad Nacional Federico Villarreal, Lima, Perú.

Departamento de Patología, Instituto Nacional de Enfermedades Neoplásicas. Lima, Perú.

Universidad de Huánuco. Huánuco, Perú.

Departamento de Diagnóstico por Imágenes, Instituto Nacional de Ciencias Neurológicas. Lima, Perú.

- Facultad de Medicina de la Universidad Nacional Mayor de San Marcos, Lima, Perú

a Residente de Neurocirugía ;b Patólogo Oncólogo; ${ }^{\circ}$ Profesor Investigador Renacyt; d Médico Neurológoradiólogo; ${ }^{\mathrm{e}}$ Docente; ${ }^{\mathrm{f}}$ Neurocirujano; ${ }^{\mathrm{g} P s i q u i a t r a ;}{ }^{\mathrm{h}}$ Tutor. 


\section{INTRODUCCIÓN}

El sarcoma fibromixoide de bajo grado es un tumor de origen mesenquimal de tejidos blandos; caracterizado por una apariencia histológica benigna, pero de agresivas características clínicas y metástasis frecuente (1). Fue descrita inicialmente por Evans en 1987 como un sarcoma raro, pero no fue hasta 1993 que él reportó una serie de 12 casos $(2,3)$. La mayoría se origina a partir del tejido celular subcutáneo, extremidades, región inguinal, pecho y pared abdominal, su presentación intracraneal es muy raramente conocida $(3,4)$. Estos tumores ocurren de manera primaria en adultos jóvenes y de mediana edad, y es aún más rara su presentación pediátrica (5). Los sarcomas son un grupo heterogéneo, representan el $1 \%$ del total de tumores del ser humano; los sarcomas primarios del SNC son raros, representan solamente el $0.7 \%$ de todos los sarcomas con una incidencia estimada porcentual de 3 por 10 millones de personas al año (6). En 2019 se reportó el caso de un paciente pediátrico en el Hospital Pediátrico Universitario de Olguín en Cuba, siendo la primera evidencia científica a nivel de Latinoamérica (7).

El origen celular y la etiología de los tumores sarcomatosos primarios del SNC son controversiales. La teoría más ampliamente aceptada indica que las células pluripotenciales mesenquimales primitivas de la dura madre, las leptomeninges y su extensión intraparenquimal, la tela coroidea, vasos sanguíneos y el estroma de los plexos coroideos como posibles candidatos(6).
A continuación describimos el caso de una paciente mujer, sus características clínicas, radiológicas, el tratamiento y finalmente la histopatología obtenida a partir de la pieza tumoral.

\section{Caso clínico}

Paciente mujer de 59 años de edad, sin antecedentes de importancia. Inició cuadro 18 meses antes de ingreso caracterizado por deterioro cognitivo progresivo (trastornos en la codificación de la memoria episódica verbal y de la memoria retrógrada, juicio inadecuado, atención dispersa y trastornos en la articulación de las palabras), cefalea opresiva fronto parietal que se incrementó con el curso de los meses asociado a signos de focalización (hemiparesia izquierda leve) por lo que acude por emergencia; en la imagenología por tomografía se halló tumoración de la convexidad parietal derecha de $2 \times 3 \mathrm{~cm}$ (figura 1), se decide estabilizar a la paciente y hacer seguimiento de la lesión por consultorio; reingresando por emergencia seis meses después por caída de nivel, hemiparesia izquierda severa, asociado a náuseas; en el estudio laboratorial general que incluyó conteo y química sanguínea dentro de parámetros normales, radiografía torácica que no mostró anormalidades.

Se solicitó tomografía computarizada (TC) contrastada en la cual se evidenció tumoración bien delimitada homogénea parietal derecha de $4 \times 5 \mathrm{~cm}$, que realza a la administración de contraste, con presencia de edema vasogénico perilesional extenso. En resonancia magnética se halló en T1 masa homogénea extraaxial hipocaptante en región parietal derecha,

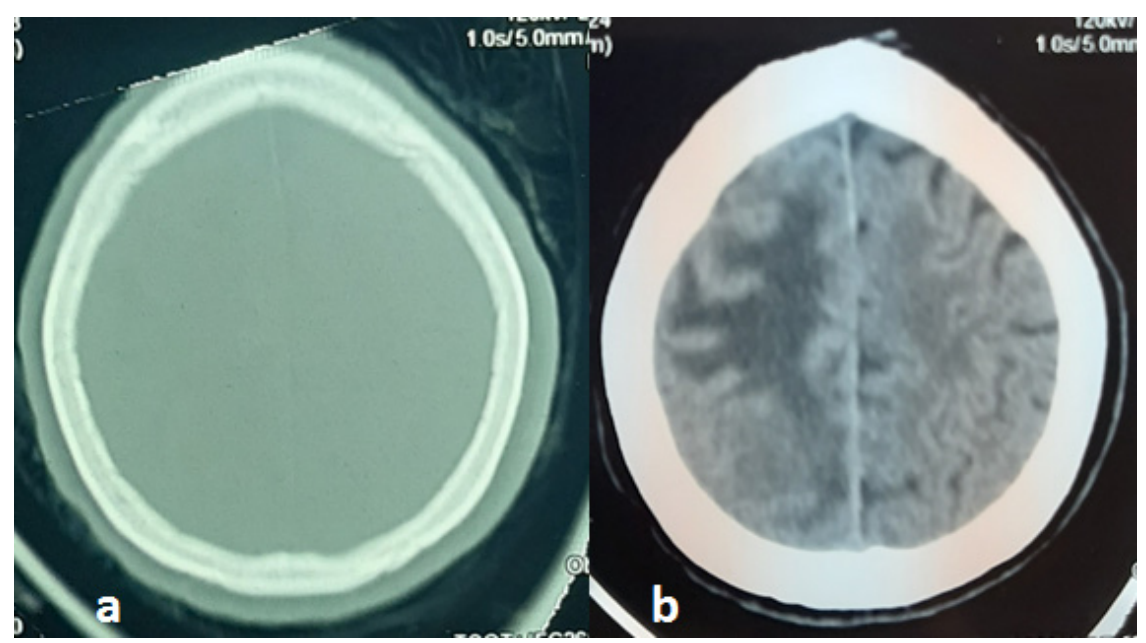

Figura 1. a) TEM cerebral sin contraste. En este estudio no se evidencia alteración ósea de bóveda craneal. b) En ventana parenquimal se aprecia edema vasogénico e imagen ovoidea isointensa en región parietal derecha. 


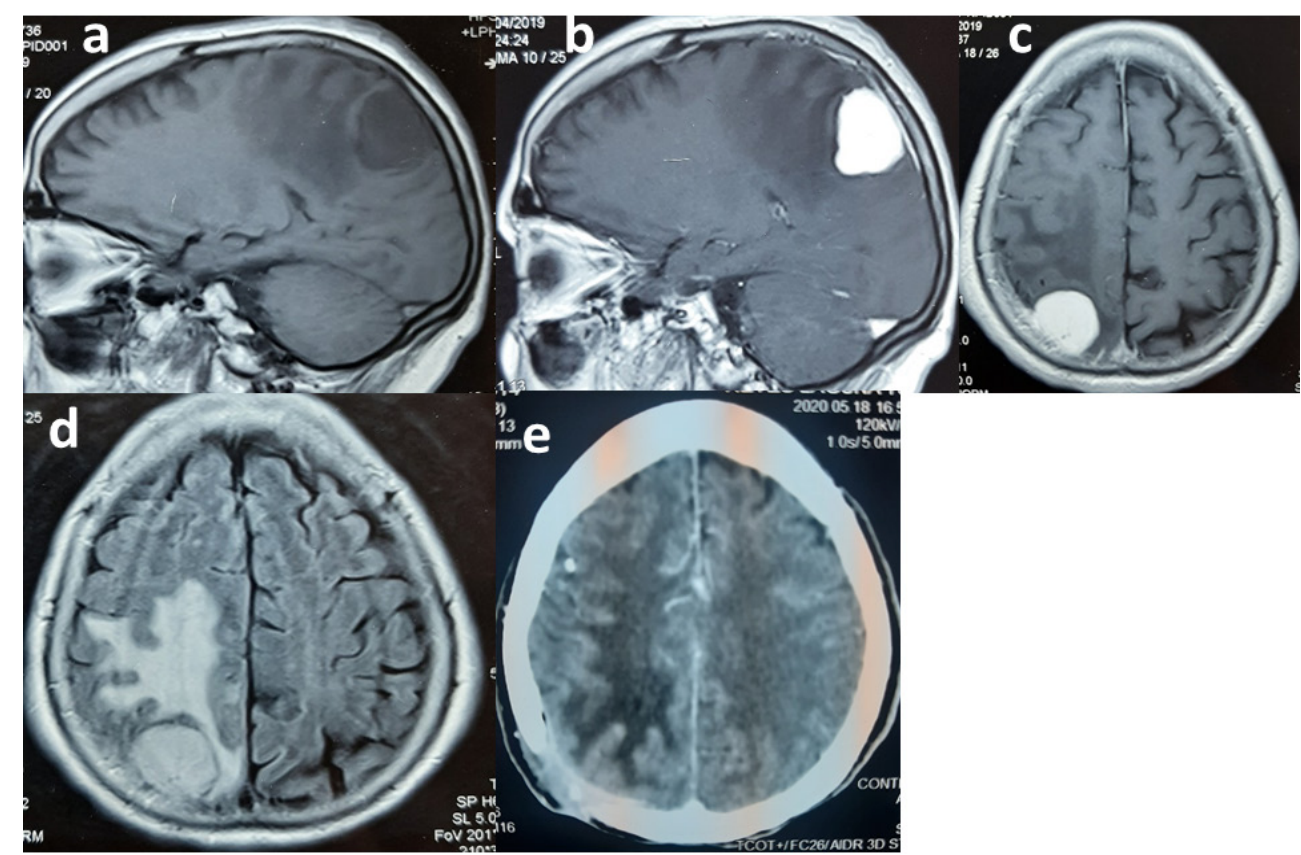

Figura 2. Resonancia Magnética. a) Secuencia T1 sagital: se observa imagen ovalada hipointensa de aproximadamente 20 × $30 \mathrm{~mm}$, asociado a edema vasogénico circundante localizado en la región cortico subcortical en parietal derecho. b) Secuencia T1, sagital con contraste: se observa la imagen ovalada se realza con el contraste de manera homogénea y de bordes definidos. c) Secuencia T1, axial con contraste: se observa imagen ovalada, se realza con el contraste de manera homogénea y de bordes definidos, en este plano se observa leve realce meníngeo adyacente a la lesión. d) Secuencia Flair, axial: se observa imagen ovalada hiperintensa de manera homogénea y de bordes definidos, asociado a edema vasogénico circundante. e) Tomografía cerebral con gadolinio control: Parénquima cerebral sin signos de recidiva.

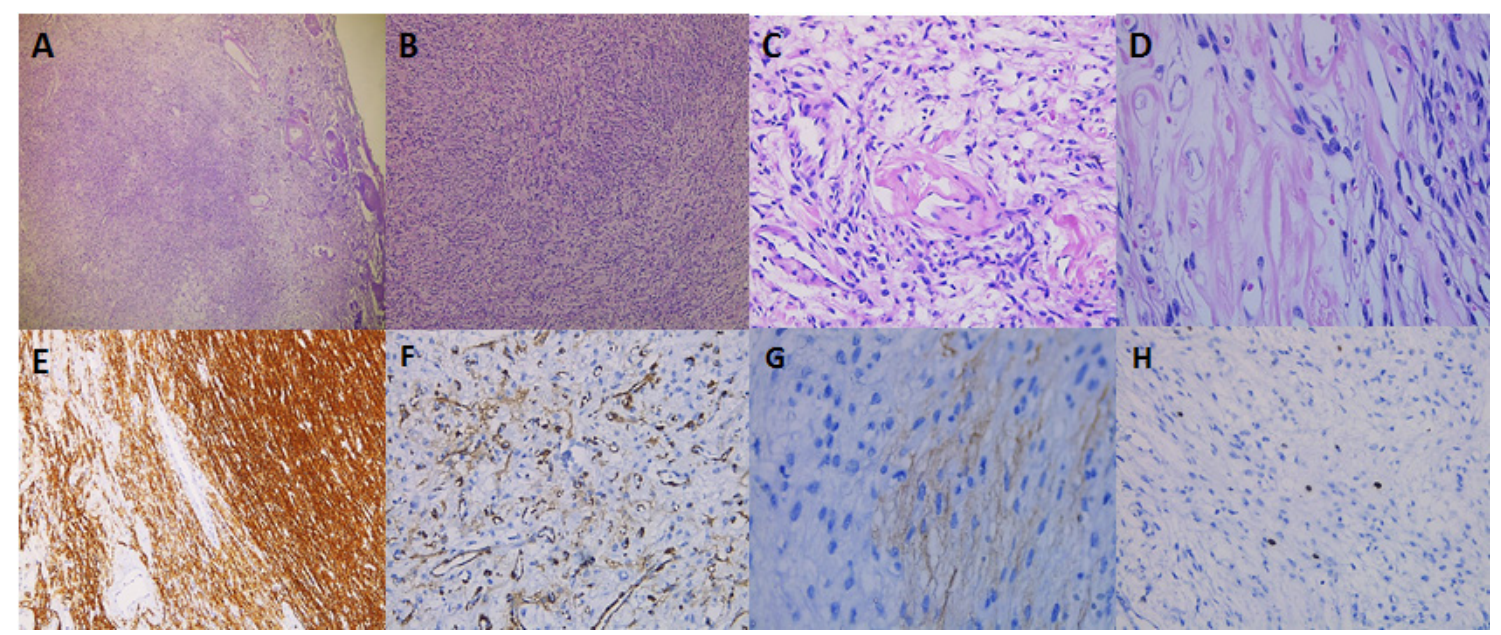

Figura 3. Descripción de hallazgos histopatológicos del tumor. A) Tumor mesenquimal intracraneal con celularidad baja a moderada, sólido y de aspecto predominantemente expansivo, observado con tinción de H\&E (Hematoxilina y Eosina). Objetivo panorámico de 2,5x (25 aumentos). B) El tumor tiene áreas de patrón arremolinado fusocelular (Tinción de H\&E, objetivo de 5x). Las mitosis son difíciles de encontrar.C) Se observan áreas de aspecto mixoide y discreta hialinización vascular (Tinción de H\&E, objetivo de 20x). D) Aspecto bifásico que alterna con áreas hipocelulares y hialinización estromal (Tinción de H\&E, objetivo de 40x). E) Estudio de inmunohistoquímica para MUC-4, que muestra tinción intensa y difusa citoplasmática de las células tumorales, (tinción marrón de peroxidasa-DAB, objetivo de 10x). F) Inmunotinción de actina músculo liso que realza el patrón vascular curvilíneo y tiñe débilmente algunas células tumorales en patrón citoplasmático, (tinción marrón de peroxidasa-DAB, objetivo de 20x). G) Tinción citoplasmática focal y débil para EMA (siglas en Inglés de antígeno de membrana epitelial, tinción marrón de peroxidasa-DAB, objetivo de 40x). H) Índice proliferativo menor de 5\% evaluado con Ki67 por inmunohistoquímica (antígeno MIB.1, tinción nuclear marrón de peroxidasa-DAB, objetivo de 20x). 
con realce al contraste; en secuencia T2 y FLAIR se evidenció marcado edema vasogénico perilesional con masa hiperintensa (figura 2), y en la Espectroscopía se apreció niveles bajos de colina y altos en N-Acetil Aspartato; paciente ingresó a sala de operaciones bajo la sospecha de un meningioma de la convexidad. Se realizó una craneotomía parietal derecha en "herradura", la tumoración tenía una invasión parcial al parénquima cerebral e infiltraba a la duramadre, se realizó su resección total y se reparó la duramadre con parche sintético.

El examen microscópico de la lesión mostró una proliferación mesenquimal fusiforme, discreta a moderadamente celular con áreas de hialinización estromal y áreas laxas de aspecto mixoide en donde identificamos estructuras vasculares curvilíneas realzadas a la inmunotinción para CD34 y Actina músculo liso, que es expresada en escasas células tumorales. El EMA es expresado débil y focalmente. La inmunotinción para MUC-4 es intensa y difusamente positiva, y el índice proliferativo evaluado con ki67, es menor de 5\%. Las mitosis son difíciles de encontrar. Las inmunotinciones para Beta Catenina, desmina, calponina, CD117, DOG1, receptor de progesterona, SOX 10, S100 y GFAP, son negativas (figura 3).

Se realizó un control tomográfico postoperatorio a las 72 horas evidenciándose exéresis completa de la lesión con presencia de edema perilesional. Posterior a la cirugía paciente fue derivada al servicio de Oncología, donde se decidió inicio de radioterapia. Actualmente un seguimiento imagenológico de la paciente a cinco meses de su cirugía no reveló indicios de lesión recidivante (figura 2 (e)).

\section{DISCUSIÓN}

Los sarcomas intracraneales primarios son patologías raras del ser humano, su estirpe fibromixoide de bajo grado viene siendo aún más inusual. Como señala Paulus et al., en su revisión de la histopatología de sarcomas primarios del SNC de 1991, hallaron 19 tumores sarcomatosos primarios de un total de 25 000 biopsias de tumores cerebrales hasta esa fecha; hallando en su estudio los subtipos de histiocitoma maligno fibroso en seis de ellos, leiomioma en tres, rabdomiosarcoma en dos, angiosarcoma en dos y solo un caso de sarcoma fibromixoide de bajo grado(8); asimismo Shimizu et al., mencionan que se han reportado los casos de sarcomas fibromixoides de bajo grado viscerales en pulmón, corazón e hígado. En cuanto a la variante intracraneal reporta solamente 8 casos, estos pacientes tenían un rango de edad entre 5 y 45 años con una mediana de 26,6 años, de ellos 7 fueron de sexo femenino y solo 1 masculino; presentándose a nivel del lóbulo frontal, temporal o ángulo pontocerebeloso, siendo la región frontal la más afectada (3 de 8) (1). Recientemente se han reportado 2 casos nuevos de pacientes de 17 y 33 años respectivamente, de los cuales 1 era una recurrencia, con lesiones a nivel de la base de cráneo (10), estos tumores son conocidos por su recurrencia local frecuente a pesar de su apariencia histológica benigna(4).

En RM hallamos una masa de aspecto sólido, hipointenso en $\mathrm{T} 1$ respecto al parénquima cerebral con captación homogénea al contraste con realce de dura adyacente (cola dural); hiperintenso y homogéneo en T2 así como en FLAIR asociado a extenso edema vasogénico perilesional, contrastado con la literatura Ballesteros et al., en su reporte informa que presentó en RM una isoseñal homogénea en T1 respecto al córtex cerebral, mientras que en T2 mostraba un aspecto más heterogéneo, era fundamentalmente hipointensa y realzaba homogéneamente tras la administración de contraste. Por lo que inicialmente se ingresó con la premisa de ser un meningioma atípico, debido a la presencia de ese gran edema tan peculiar. "Los sarcomas intracraneales primarios son tumores extremadamente raros y la mayoría son fibrosarcomas. No existen rasgos radiológicos definitivos que diferencien el sarcoma fibromixoide de bajo grado de otro tipo de fibrosarcomas, ya que se comportan como masas sólidas con grados variables de realce, necrosis o degeneración quística y hemorrágica"(3). Aunque son raros forman parte del diferencial de las lesiones con base dural (1).

En la inmunohistopatología, evidenciamos realce en la inmunotinción para CD34, Actina de músculo liso y MUC-4; como bien lo describe Buccoliero et al., en su estudio, menciona que puede haber reactividad para CD34 en más del 30\% de los casos; asimismo menciona que los mixofibrosarcomas comprenden un amplio espectro histológico, desde una apariencia hipocelular benigna hasta tumores pleomórficos de alto grado (6).

El tratamiento de elección sigue siendo la escisión quirúrgica, debido a su rareza en el SNC las recomendaciones actuales para realizar terapia adyuvante para lesiones intracraneales sigue siendo poco claro $(1,4)$. Asimismo Saito et al., menciona que la cirugía, además, es el tratamiento de las recidivas 
y metástasis en esta patología; además reportó que la radiocirugía asociado a radioterapia contribuyó al control tumoral en su casuística, por lo tanto la radiación podría ser una herramienta en el manejo de los tumores sarcomatosos fibromixoides de bajo grado de origen intracraneal( $(9)$.

La paciente del presente reporte, no presenta evidencia imagenológica de recidiva tumoral hasta la fecha.

El sarcoma fibromixoide de bajo grado intracraneal es una patología infrecuente, de curso clínico e histología aparentemente benignos, pero con comportamiento local y a distancia agresivos. El diagnóstico definitivo lo da la histopatología de la pieza tumoral extraída. La poca evidencia que se tiene a la fecha no permite conocer a mayor detalle a esta entidad nosológica, sin embargo cada estudio adicional que se obtenga contribuirá al mejor entendimiento de esta.

\section{Correspondencia:}

José Luis León Palacios,

Av. Honorio Delgado 210 San Martín de Porres- Lima, Correo electrónico: jose.leon.p@upch.pe

Declaración de financiamiento y de conflictos de intereses: El reporte fue financiado por los autores; declaran que no existe conflicto de interés.

\section{REFERENCIAS BIBLIOGRAFICAS}

1. Shimizu Y, Tsuchiya K, Fujisawa H. Intracranial Low-Grade Fibromyxoid Sarcoma: Findings on Electron Microscopy and Histologic Analysis. World Neurosurg. 2020;135:301-5.

2. Chen N, Gong J, Nie L, Chen X, Xu M, Chen M, et al. Primary intracranial low-grade fibromyxoid sarcoma with FUS gene rearrangement. Neuropathol Off J Jpn Soc Neuropathol. 2015;35(4):348-53.
3. Ballesteros E, Casalots J, Rovira A, Prenafeta M. Sarcoma fibromixoide de bajo grado intracraneal: a propósito de un caso. Radiología. 2011;53(2):171-4.

4. Karthigeyan M, Malik P, Garg S, Radotra BD, Salunke P, Sunil N, et al. Intracranial low-grade fibromyxoid sarcoma with atypical radiology. World Neurosurg. 2019;122:500-4.

5. White IK, Scherer AG, Baumanis MM,Abdulkader M, Fulkerson DH. Rapidly enlarging low-grade fibromyxoid sarcoma with intracranial extension in a 5-year-old girl: case report. J Neurosurg Pediatr. 2015;16(4):372-6.

6. Buccoliero AM, Castiglione F, Garbini F, Degl'Innocenti DR, Moncini D, Franchi A, et al. Primary cerebral myxofibrosarcoma: clinical, morphologic, immunohistochemical, molecular, and ultrastructural study of an infrequent tumor in an extraordinary localization. J Pediatr Hematol Oncol. 2011;33(7):e279.

7. Meriño YFR, Cruz ABA, Santiesteban MZ, Jiménez PAD, Sanamé DAM, Batista RAM. Tumor cerebral maligno epitelial-mesenquimal de probable origen neuroectodérmico sarcomatoso. : 11 .

8. Paulus W, Slowik F, Jellinger K. Primary intracranial sarcomas: histopathological features of 19 cases. Histopathology. 1991;18(5):395-402.

9. Saito R, Kumabe T, Watanabe M, Jokura H, Shibuya M, Nakazato Y, et al. Low-grade fibromyxoid sarcoma of intracranial origin. J Neurosurg. 2008;108(4):798802.

10. Chetverikova E, Kasenõmm P. Low-Grade Fibromyxoid Sarcoma of the Lateral Skull Base: Presentation of Two Cases. Case Rep Otolaryngol. 8 de julio de 2019; 2019:1-6.

\begin{tabular}{l}
\hline Recibido: 10/06/2020 \\
Aceptado: 13/09/2020 \\
\hline
\end{tabular}

